\title{
Efficacy and safety of phosphodieterase-5 inhibitors for the treatment of erectile dysfunction secondary to spinal cord injury
}

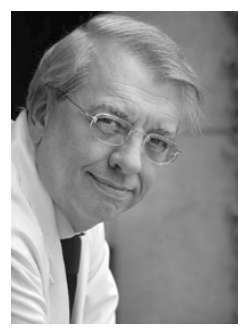

\author{
JJ Wyndaele \\ Antwerp, Belgium \\ E-mail: wyndaelejj@skynet.be
}

I hope to see many of you during the ISCOS Annual Scientific Meeting in Vienna on 14-16 September. It is the yearly event where we discuss editing and publication matters and can have direct communication between authors, reviewers and board members.

As previously mentioned, there is now a longer list of publications available online but waiting to be printed. This is due to a much higher number of submissions, and despite an increase in rejection rate. Some patience is needed while we consider if the print backlog can be eventually shortened. Meanwhile, the accepted papers are available in search engines, can be read and studied, and can be referenced.

Review: Jia et al. assessed the efficacy and safety of phosphodieterase-5 inhibitors on erectile dysfunction secondary to SCI. Six randomized controlled trials showed that, compared with placebo, PDE5 inhibitors intake was associated with significant improvements. Despite significant greater incidences of some adverse events, most were mild to moderate and transient. The review concludes that PDE5 inhibitors are effective and well tolerated and could be considered as the first choice of treatment for erectile dysfunction after SCI.

Animal Studies: Graham et al. determined the effects of SCI on aspects of the focal adhesion kinase (FAK) signalling pathway 56 days post-injury in rat gastrocnemius. Diminished FAK signalling was found. SCI had no effect on mRNA levels for genes encoding components of the focal adhesion. Loading the cytoskeleton of skeletal muscle as soon as possible following SCI may be a way to prevent muscle atrophy through activation of the integrin/FAK pathway. Zhou et al. found that Calcitriol promotes locomotor recovery after SCI through its impacts of depression of oxidative stress and inhibition of apoptosis, as well as promotion of autophagy. Kjell et al. were confronted with altered health status of animals they planned to use for their research. But this led to the finding that virus infections may have positive effects on functional recovery after SCI: RPV-1a infection may be neuroprotective by dampening secondary damage. Hayashibi et al. found that forced treadmill training by plantar placement (PP), as compared with dorsal placement (DP), of the dorsal paws promoted the behavioural and histological improvement of rats with SCI.

Pressure ulcers: Joseph and Nillson-Wikmar report that pressure ulcers and pulmonary complications were prevalent during specialized acute phase after SCI in South Africa. Special attention for persons with motor complete lesions and those with vertebral injuries is again stressed.

Functional measures, datasets: Bea-Munoz et al. analysed websites related to SCI and available in Spanish to be of medium quality. Reliability of the contents, readability, inclusion of more quality labels is mandatory. It shows again how web information needs to be read and interpreted with caution. Slavin et al. found, in a large sample, Paediatric Spinal Cord Injury Activity Measure to exhibit strong psychometric properties for children with tetraplegia. Replenishment of the General Mobility and Daily Routines item banks is needed to reduce ceiling effects noted for youth with paraplegia. Scivoletto et al. found a good validity of the Italian version of the SCIM III SR Post et al. developed the International SCI Activities and Participation (A\&P) Basic Data Set.

The editorial office is always available for correspondence, ideas, suggestions and comments: Spinalcord@uza.be 